\title{
The fundamental constants of physics and the International System of Units
}

\author{
Diederik Sybolt Wiersma ${ }^{1,2}$ (]) Giovanni Mana ${ }^{1,3}$
}

Received: 7 June 2021 / Accepted: 12 September 2021 / Published online: 19 October 2021

(c) The Author(s) 2021

\begin{abstract}
Air Canada managed to have a passenger aircraft run out of fuel in mid-air due to confusion about metric units (Stephenson in Mars climate orbiter mishap investigation board phase I report, NASA, 1999), and NASA lost an entire spacecraft due to a misunderstanding amongst engineers about the units used in the propulsion system design (Witkin in Jet's fuel ran out after metric conversion errors, The New York Times, 1983). Measurements only make sense if the units are correct and well-defined. A unit of measurement is a definite magnitude of a quantity, defined by convention or law. Any other quantity of that kind can then be expressed as a multiple or submultiple of the unit of measurement. The Egyptians used the Farao as definite magnitude, while many years later, the french revolutionists introduced the earth as a reference and laid the foundations for the modern decimal system. Since recently, we have a truly universal and stable system that uses physics's natural constants and laws to define the base units of measurement. This paper explains how this new concept works and how it is implemented in practice.
\end{abstract}

Keywords Fundamental constants $\cdot$ International system of units $\cdot$ Metrology

Diederik Sybolt Wiersma and Giovanni Mana contributed equally to the manuscript.

This paper celebrates Professor Gustavo Colonnetti (Turin, 8 November 1886 - Turin, 20 March 1968), who was elected member of the Accademia Nazionale dei Lincei in 1947. He was a mathematician and engineer, who made contributions to continuum mechanics, and a teacher aiming to high culture and only subordinately to the professional preparation. He was rector of the Politecnico di Torino and president of the Consiglio Nazionale delle Ricerche. In the fifties of the last century, he triggered the development of Italian metrology through the foundation of the Istituto Dinamometrico and Istituto Termometrico that, in 1968, merged in the Istituto di Metrologia "G Colonnetti", now Istituto Nazionale di Ricerca Metrologica.

Diederik Sybolt Wiersma

d.wiersma@inrim.it; diederik.wiersma@unifi.it

Giovanni Mana

g.mana@inrim.it; giovanni.mana@unito.it

1 INRIM-Istituto Nazionale di Ricerca Metrologica, Strada delle cacce 91, 10135 Turin, Italy

\section{Introduction}

From 20 May 2019, to allow the international system of units (the SI) to exploit both the advances in our understanding of Nature and recent technological developments, all units are implicitly defined in terms of seven fundamental constants, without distinctions between primary and derived units (Stock et al. 2019). This means that the natural constants serve as definite magnitude, which, together with the laws of physics, allow to construct a system of units of measurement that is truly universal, accessible to everyone, and that does not require physical objects or "artefacts" as reference.

The new SI is the system of units in which (Joint Committee for Guides in Metrology 2019)

2 LENS and Dipartimento di Fisica e Astronomia, UNIFI-Università di Firenze, Via Nello Carrara 1, 50019 Sesto Fiorentino, Firenze, Italy

3 Dipartimento di Fisica, UNITO-Università di Torino, via Pietro Giuria 1, 10125 Turin, Italy 
- the unperturbed ground state hyperfine transition frequency of the ${ }^{133} \mathrm{Cs}$ atom $\Delta v_{\mathrm{Cs}}$ is $9,192,631,770 \mathrm{~Hz}$,

- The speed of light in vacuum $c$ is $299,792,458 \mathrm{~m} / \mathrm{s}$,

- The Planck constant $h$ is $6.62607015 \times 10^{-34} \mathrm{~J} / \mathrm{S}$,

- The elementary charge $e$ is $1.602176634 \times 10^{-19} \mathrm{C}$,

- The Boltzmann constant $k_{\mathrm{B}}$ is $1.380649 \times 10^{-23} \mathrm{~J} / \mathrm{K}$,

- The Avogadro constant $N_{\mathrm{A}}$ is $6.02214076 \times 10^{23} \mathrm{~mol}^{-1}$,

- The luminous efficacy of monochromatic radiation of frequency $540 \times 10^{12} \mathrm{~Hz}, K_{\mathrm{cd}}$, is $683 \mathrm{~lm} / \mathrm{W}$,

where the units hertz $(\mathrm{Hz})$, joule $(\mathrm{J})$, coulomb $(\mathrm{C})$, lumen $(\mathrm{lm})$, and watt $(\mathrm{W})$ are related to the units second (s), metre $(\mathrm{m})$, kilogram (kg), ampere (A), kelvin (K), mole (mol), and candela (cd) according to $\mathrm{Hz}=\mathrm{s}^{-1}, \mathrm{~J}=\mathrm{kg} \mathrm{m}^{2} \mathrm{~s}^{-2}, \mathrm{C}=\mathrm{A}$ $\mathrm{s}, \mathrm{lm}=\mathrm{cd} \mathrm{m}^{2} \mathrm{~m}^{-2}=\mathrm{cd} \mathrm{sr}$, and $\mathrm{W}=\mathrm{kg} \mathrm{m}^{2} \mathrm{~s}^{-3}$. Seven base units (metre, kilogram, second, ampere, kelvin, mole, and candela) have been chosen for historical reasons and are, by convention, regarded as dimensionally independent.

What constants to fix was determined by trading off between the depth and breadth of the theories that they tag and the need to ensure continuity to the everyday metrology, which continuity, in turn, correlates to how well we are able to measure them. Their values have been conventionally chosen by agreement to minimise the differences between the sizes of the new units and the old ones (Mohr et al. 2018), which took a considerable technical effort of the metrology community before the redefinition in 2019 could take place. The new definitions will therefore have no direct effect on our daily lives, while at the same time, they set the stage for a significant improvement of measurement accuracy. By using the natural constants as references, all future technological developments are automatically built into the system.

In this paper we show how the constants' definitions and units depend on the measurement technologies and the theoretical framework used to explain the experimental observations.

\section{Fundamental constants}

Any universal fundamental constant may be described as a concept synthesizer expressing the unification of two previously unconnected physical concepts into a single one of extended validity, Lévy-Leblond (1977).

A numerical measure is not enough to endow a quantity with existence. It must also have a mathematical counterpart and be part of a network of quantities and relationships expressed by a theory supplying the operational definitions associating mathematical concepts and measurement results.

The unification of theories implies new mathematical laws that reduce the number of free parameters and supply conversion factors via constants establishing relationships between quantities that previously took different realities. Examples of these conversion factors are

- The speed light in vacuum $c$ that by Maxwell's equations is related to the electric permittivity $\epsilon_{0}$ and magnetic permeability $\mu_{0}$ of the vacuum by $c=1 / \sqrt{\epsilon_{0} \mu_{0}}$;

- The magnetic permeability of the vacuum $\mu_{0}$ that by Maxwell's equations is related to the magnetic $\mathbf{H}$ and electric fields $\mathbf{E}$ in a vacuum by $\nabla \times \mathbf{E}=-\mu_{0} \partial_{t} \mathbf{H}$;

- The speed light in vacuum $c$ that through general relativity is related to the energy $E$ and mass $m$ by $E=m c^{2}$;

- The Planck constant $h$ that Einstein related to the frequency $v$ and energy $E$ of a photon by $E=h v$;

- The Joule constant $J$ that relates heat $Q$ and energy flow $E$ by $Q=J E$;

- The Boltzmann constant $k_{\mathrm{B}}$ that relates the thermodynamical entropy $H$ and Shannon information $S$ by $H=\ln (2) k_{\mathrm{B}} S$;

- The Avogadro constant $N_{\mathrm{A}}$ that relates the number of basic amounts that take part in chemical reactions $N$ and the amount-of-substance $n$ by $N=N_{\mathrm{A}} n$;

- The Hubble constant $H_{0}$ that tells how fast the universe is expanding and relates the recession velocity $v$ to the distance $d$ of the observed points by $v=H_{0} d$.

The recognition that fundamental constants underpin unification decreases as the novelty fades away. Eventually, many of them disappear, and what remains are conversion factors that enable physical quantities to be expressed in different units. We can select the values of these conversion factors by conceptual and mathematical simplicity, but, in practice, they are constrained by technological convenience.

A never born constant relates inertia and gravitational charge. In definition 1 of the Principia, Newton (1689) writes "... [inertia] is proportional to the weight, as I have found by experiments on pendulums, very accurately made, which shall be shown hereafter". This identity is an intrinsic feature of general relativity, which explains the motion in a gravitational field as inertial motion in curved spacetime.

A constant that is no longer needed is the mechanical equivalent of heat, which highlights that heat is an energy flow (Greenslade 1980). It was not until the middle of the 19th century that thermodynamics related heat and energy, which had previously been defined independently and measured by different units. We are now fully accustomed to this identification which is embedded into the background of physics. For this reason, no conceptual role is given to the Joule constant anymore and it is never explicitly written into equations.

The Boltzmann and Avogadro constants still survive, but the second is no more considered to be fundamental. The first stems from the merging of thermodynamics and 
statistics and expresses the fact that the thermodynamical entropy is a measure of the Shannon information given the probability distribution (at equilibrium) of a system being in any cell of its phase space.

The Avogadro constant stands at the union of chemistry and physics (Milton 2011; Massa and Mana 2016; Güttler et al. 2019). It originated from the need to measure the behaviour of matter in chemical reactions (Milton and Mills 2009). The concept of an amount-of-substance lies in the law of constant proportions: a pure compound always contains the same elements in the same mass ratio. This law played a fundamental part in the development of chemistry: the amount of an element or compound can be traced back to the amount of a reference-e.g., $12 \mathrm{~g}$ of ${ }^{12} \mathrm{C}$ - by chains of stoichiometric proportions. In the nineteenth century, investigations of several chemists revealed that the amount of substance always comes in integer quanta, basic amounts that take part in chemical reactions. They found these quanta universal, e.g., independent of time, place and thermodynamic state. This strengthened the atomic hypothesis and linked the vindication of the existence of atoms to the determination of the number of quanta in a mole, a constant introduced by Perrin (1909) and named after Avogadro.

\section{Mechanical quantities}

\subsection{Space and time}

\subsubsection{Newton}

Newton (1689) embedded mechanics into a three-dimensional Euclidean space with an added time parameter. At the beginning of the Principia, in a Scholium, he lays out definitions of time and space and sets out to define practical realizations of their units

- Absolute, true, and mathematical time, of itself, and from its own nature, flows equably without relation to anything external, and by another name is called duration. Relative, apparent, and common time, is some sensible and external (whether accurate or unequable) measure of duration by the means of motion, which is commonly used instead of true time; such as an hour, a day, a month, a year.

- Absolute space, in its own nature, without relation to anything external, remains always similar and immovable. Relative space is some movable dimension or measure of the absolute spaces; which our senses determine by its position to bodies; and which is commonly taken for immovable space; such is the dimension of a subterraneous, an aerial, or celestial space, determined by its position in respect of the earth.
Without a predetermined reference frame and a clock, the only observable quantities are relative distances. Actually, they are angles (by reference to the celestial mechanics), but this does not change the problem. Therefore, given $p$ ordered sets of relative distances (without any time coordinate) of a Newtonian system of $N$ gravitating bodies, the problem, first solved by Tait (1884) for the simplest case of free bodies, is to create a reference frame and a clock consistent with Newtonian mechanics.

Each set of distances is assumed to have the same Newtonian time. Also, an invariant distance-i.e., a practical realization of the length unit-is assumed in each set, for instance, the distance between two bodies. There is no way to test these hypotheses a priori because no external space and time references exist. They are dictated by the model explaining the data and will be supported (or falsified) a posteriori by over-determining the model parameters.

Without a predetermined reference, the input data are $m=N(N-1) / 2$ relative distances. The unknowns are $n=6 N+p-12$ because the origin, orientation, and velocity of the reference frame, the epoch of a data set, and space and time units (which do not change the relative distances and velocities) are chosen freely. Provided that $m p>n$, we can solve the problem; actually, it is over-determined, and consistency vindicates the Newtonian theory.

In the Newtonian universe of gravitating bodies, length is the primary quantity, while time is derived from motion. As shown by Barbour (2001, 2009), it is an abstraction facilitating the description of motion, and it is built by fitting the Newtonian model to all the bodies' relative distances.

An approximation is the ephemeris time, which results from fitting Newtonian dynamics to the solar system. Until 1967, the second (equal to the fraction 1/31556925.9747 of the tropical year for 1900 January 0 at 12 hours ephemeris time) was realized from measurements of the positions (ephemerides) of astronomical objects using a dynamical model of their motion. Accordingly, a clock is "a mechanism for measuring the time that is continually synchronized as nearly as may be with ephemeris time" (Clemence 1957).

In the simplest case of free bodies, the Newton time is the varying distance between the two bodies. Since it is independent of the chosen motionless origin of the reference frame, it is independent of the inertial frame. In practice, distances in Newtonian (celestial) mechanics are not observable to a significant level of accuracy. Therefore time and space units were defined independently, as is obvious from the set of successive definitions of the meter.

Furthermore, the second has to be defined for the tropical year "at a given time" (1900 January 0 at $12 \mathrm{~h}$ ) because the orbit of the Earth and the reference point that one can choose to define a tropical year are not immutable. Formulas are adjusted to centuries of observations and would change with new, more accurate ones. Another problem is that it requires 
years of observations to realize the second at a given time with uncertainty not too far with that implied in the definition, e.g., $10^{-10} \mathrm{~s}$.

This approach came up when Maxwell's electromagnetic theory appeared and when the associated practical and theoretical developments would inevitably yield a new theory to describe space and time. As we shall see in the next section, space and time units then shift from independent to completely related.

\subsubsection{Einstein}

In the framework of relativistic dynamics, the construction of space and time proceeds differently. Clocks are supposed to be available everywhere they are needed: they are isolated systems where an event repeats cyclically. This definition leaves aside what time is, apart from it resulting from the counting of cycles. Furthermore, without a predetermined external clock, we cannot say that the cycle duration is constant-i.e. that one second today is equal to one second tomorrow. Hence, we assume it on a theoretical basis and vindicate it a posteriori by fitting a theoretical framework to redundant data.

Without clock synchronization and a predetermined ruler, the only available data are time intervals, as measured by the same clock. Considering free-bodies again, the distance between $A$ and $B$ is half the time interval, measured by the A's clock, between the transits to A of a photon sent from A and reflected by B. Also, it is associated with the arithmeticmean of the photon transits to A. Clock synchronization is carried out by imposing that the reflection at B occurred at the arithmetic mean of the transits to A. This procedure allows synchronising and calibrating clocks relatively at rest and building a Euclidean reference frame and a universal time scale.

While the Newtonian space-time foliates in a unique stack of three-dimensional Euclidean leaves of simultaneous events, the foliation of the Minkowski space-time depends on clock velocities. In fact, the assumptions of space and time homogeneity and isotropy (no location, time, and direction are preferred) and the principle of relativity (the laws of Nature are independent of the reference frame) imply Lorentz transformations between inertial frames (were free bodies move in straight lines at constant speed).

The foliation of a gravitating space-time is not always globally possible. The usual solution is to choose a given coordinate system and its associated metric and to replace Einstein synchronization with coordinate synchronization (Soffel et al. 2003). Times-of-flight still give distances, but they are not strictly measurements of a space interval. Rather, they are proper times from which space coordinates can be derived for the events "photon transit at the B clock".

\subsubsection{Mise an pratique of the metre}

The relativistic definition of distances shows that the speed of light is the conversion factor between the measurement units used for space and time intervals. It can be set to unity for mathematical simplicity and to emphasize the interplay of space and time coordinates.

This conversion-factor role is played in expressing astronomical distances in light-year; as defined by the International Astronomical Union, the distance that light travels in vacuum in one Julian year. In 1838, Bessel firstly used the year to express an astronomical distance: he reported his measured distance of the binary star 61 Cygni as 10.3 light-years (??). Providing the time it takes for light to reach Earth encodes that we see into the past and that telescopes are past-travelling machines.

A workable choice of the $c$ value for the everyday metrology is obtained by assigning it a dimensional value and using different quantities and units to represent (to some level of uncertainty) space and time in a non-relativistic world (a set of foliations of the Minkowski space-time having a low speed to one another). The stipulated $c$ value specifies the time-of-flight that corresponds to one metre. Hence, length measurements determine time-of-flight, either directly or indirectly, via interferometry and the $\lambda=c / v$ relationship between wavelength and frequency of a plane wave in a vacuum. In this last case, the measurement of the frequency $v$ in terms of $\Delta v_{\mathrm{Cs}}$ is an essential step.

\subsection{Mass}

\subsubsection{Classical physics}

In both classical and relativistic dynamics, (inertial) mass is a property needed to characterize the dynamical behaviour of interacting systems. If we take the speed of light to be unity and dimensionless, the relativistically invariant mass is the internal energy of the system (the total energy in the system's rest frame).

The additivity of its mathematical counterpart can be used to trace mass measurements back to a subsystem, which we agree to use as a standard. The atomic theory provides the framework to base mass metrology on the masses of atoms. If an atom is chosen as a mass standard, mass measurements can be traced back to atom counting. To bring a macroscopic count into practice, a ${ }^{28} \mathrm{Si}$ monocrystal is shaped as a quasi-perfect ball; the number $N_{\mathrm{Si}}$ of atoms in it is obtained from the measurement of the volume $V$ and lattice parameter $a_{0}$ according to $8 V / a_{0}^{3}$, where $a_{0}^{3} / 8$ is the atom volume and 8 is the number of atoms in the cubic unit cell (Bettin et al. 2013).

Since ${ }^{28} \mathrm{Si}$ crystals are never mono-isotopic, the amount of substance fraction of $\mathrm{Si}$ isotopes is measured by absolute 
mass-spectrometry. Furthermore, they may contain impurities, interstitial atoms, and vacancies, which would imply that the number of lattice nodes does not correspond to the number of $\mathrm{Si}$ atoms. This means that crystals must be characterized both structurally and chemically to apply the appropriate corrections. The mass, thickness, and chemical composition of the surface layer (mainly $\mathrm{SiO}_{2}$ ) must also be considered; they are measured by optical and X-ray spectroscopy and reflectometry.

Eventually, we must include the interaction and kinetic energies of the sub-systems. The formation enthalpy of a silicon crystal (the energy lost by one mole of a dilute gas when it forms a crystal at absolute zero temperature) is about $450 \mathrm{~kJ} / \mathrm{mol}$ (Cox et al. 1989). This means that the binding energy contribution to the mass is $5 \mathrm{ng} / \mathrm{mol}$ or $0.2 \mathrm{ng} / \mathrm{g}$, which is negligible at the present level of accuracy (Davis and Milton 2014).

According to the Dulong and Petit law, the molar heat capacity of a crystal is $3 R$, where $R=8.314 \mathrm{~J} / \mathrm{K} / \mathrm{mol}$ is the gas constant. Therefore, in the case of a silicon crystal at room temperature, the thermal energy contribution to mass is $75 \mathrm{pg} / \mathrm{mol}$ or $2.7 \mathrm{pg} / \mathrm{g}$, in relative terms, which is also negligible.

\subsubsection{Quantum physics}

According to the Planck equation $E=h v$, the energy $E$ of a stationary quantum system is proportional to the frequency $v$ of its quantum-mechanical wave function. Consequently, the Planck constant $h$ converts energy units into frequency units. Additionally, when quantum mechanics is combined with relativity, $h$ converts the (Compton) frequency of the relativistic wave (in the reference frame where it is at rest) to the system mass-energy $E=m c^{2}$. In fact, combining the Planck and Einstein equations, we obtain $h v=m c^{2}$, which relates frequency and mass, and shows that $h / c^{2}$ converts between frequency and mass units. This equation indicates that if we set $c=h=1$, length and time are measured in seconds and mass in hertz. This choice highlights the unification achieved by relativistic quantum mechanics.

We have seen that Newton's and Einstein's space and time are shortcuts to describe the inner relationships of a physical system by building an external framework to place it in. This outer reference frame is made on only internal data, but a clock was assumed to be associated with each particle in the system. Quantum mechanics supplies the otherwise missing operational definition of this clock by identifying it with the particle itself via its Compton's frequency (Lan et al. 2013; Müller 2014).

The international system of units fixes the frequency $\Delta v_{\mathrm{Cs}}$ of the photon emitted in the transition between two quantum states of the caesium 133 atom and values of the speed of light and Planck constant. Therefore, neglecting the recoil, the difference between the masses of the ${ }^{133} \mathrm{Cs}$ atom in the excited and ground states is exactly $h \Delta v_{\mathrm{Cs}} / c^{2}$ and realises the mass unit. The challenge is to scale this extremely tiny mass, about $7 \times 10^{-38} \mathrm{~g}$, up to a macroscopic value, to within an accuracy better than $20 \mathrm{ng} / \mathrm{g}$.

A way to do this is again to count silicon atoms (Mana and Massa 2012). In fact, recoiling ${ }^{133} \mathrm{Cs}$ or ${ }^{37} \mathrm{Rb}$ atoms by photons in an interferometer allows the ratio between their inertia and the Planck constant to be determined, and relative mass spectrometry by a Penning trap delivers the masses of the $\mathrm{Si}$ isotopes tracing they back to these atom masses.

A way of measuring $m / h$, where $m$ is a macroscopic mass, is by a Kibble balance (Kibble 1976; Schlamminger and Haddad 2019). This balance compares, indirectly, the electrical and mechanical powers required to move a mass with uniform vertical velocity against the Earth's gravity. Firstly, the balance is used to equilibrate the weight $m g$ of the mass by a force generated by the interaction between the electrical current $I$ flowing in a coil supporting it and a magnetic flux. Secondly, the mass-coil system is moved vertically in the same magnetic flux with uniform velocity $u$, and the electromotive force $\mathscr{E}$ at the coil ends is measured. By combining the forces' equilibrium with Faraday's law, we obtain the sought power equality $\mathscr{E} I=m g u$.

After some basic simplifications, the electrical power is written as $\mathscr{E} I \propto h v^{2}$, where (as will be discussed in section 5) the proportionality constant is a ratio of integers and $v$ is the frequency of the microwaves irradiating a Josephson device in the measurements of electrical power. Eventually, the mass to be measured is expressed in terms of the Planck constant, the speed of light, and $\Delta v_{\mathrm{Cs}}$ as $m \propto h v^{2} /(g u)$.

\section{Thermodynamic quantities}

Statistical mechanics links probabilities to entropy via the Boltzmann constant. Jaynes (1957) showed that it is possible to formulate equilibrium thermodynamics as a theory of statistical inference. This formulation rests on the discovery by Shannon (1948) that there is a unique measure of the missing information encoded by a discrete probability distribution. Accordingly, the microstate probabilities $p_{i}$ maximising the Shannon information and constrained by whatever is known, are the most unbiased representation of our knowledge of the state of an isolated thermodynamic system at equilibrium.

Since the Shannon information $S=-\sum_{i} p_{i} \log _{2}\left(p_{i}\right)$ (measured in bits) is related to the thermodynamical entropy by $H=k_{\mathrm{B}} \ln (2) S$, the quantum of information $k_{\mathrm{B}} \ln (2)$ convert bits to $\mathrm{J} / \mathrm{K}$ and works as a token of equilibrium-thermodynamics merging into information theory.

The stipulated $k_{\mathrm{B}}$ value specifies the amount of energy per degree of freedom that corresponds to one kelvin. Therefore, temperature measurements determine the molecular kinetic 
energy and trace it back to the speed of light and Planck constant (de Podesta 2016).

The Boltzmann constant also occurs in statistical quantum mechanics through the $k_{\mathrm{B}} \ln (2) / h$ ratio. With a continuous phase space, the information encoded in a system is infinite. This infinity is, of course, not possible. If following Rovelli (1996) we assume that the maximum readable information can localise a system only in a finite volume of its phase space, there must exist a universal constant, having the dimensions of an action, determining the minimal volume of this phase space. We can view this quantum of action as converting bits to units of action and the $k_{\mathrm{B}} \ln (2) / h$ ratio as converting units of action to $\mathrm{J} / \mathrm{K}$.

The maximal bits of (Shannon) information that an isolated thermodynamic system at equilibrium can store by exploiting all of its degrees of freedom is the Bekenstein (1981) limit $4 \pi^{2} R M c /(h \ln (2))$, where $M$ and $R$ are the system's rest mass and effective radius. Since $R$ cannot be smaller than $M$ 's horizon radius, this limit is saturated by the Bekenstein-Hawking entropy of spherical and electrically neutral black holes, $A /\left(4 \lambda_{\mathrm{p}}^{2} \ln (2)\right)$, where $A$ is the horizon area and $\lambda_{\mathrm{P}}=\sqrt{\hbar G / c^{3}}$ is the Planck length, which implies an upper bound on the information that can be stored in any given volume.

\section{Electromagnetic quantities}

The reproducibilities of the Josephson and quantum Hall effects are such that electrical measurements were traced back to them since 1990 (Keller 2018).

The Josephson effect (Josephson 1962), a quantised voltage $V=n v / K_{\mathrm{J}}$ across two superconductors separated by a thin insulating layer and irradiated by a microwave having frequency $v$, connects voltage to the elementary charge and Planck constant via the Josephson constant $K_{\mathrm{J}}=2 e / h$.

Similarly, the (integer) quantum Hall effect (plateaus of the Hall voltage of a two-dimensional electron gas at low temperature) can be used to realise quantised resistors having an electrical resistance that is a submultiple of the von Klitzing constant, $R_{\mathrm{K}}=h / e^{2}$ (von Klitzing et al. 1980; von Klitzing 2017).

Therefore, though it is not a fundamental constant as outlined in Sect. 2, defining the Planck constant made convenient also defining the elementary charge because it provides fixed values for the Josephson and von Klitzing constants. The fixed values of $h$ and $e$ in the new SI and, consequently, the fixed values of $K_{\mathrm{J}}$ and $R_{\mathrm{K}}$, incorporate the electrical quantum units into the system and replace the previous definition of the ampere.

\section{Theory testing}

If photons exist, the photoelectric effect opens a way to measure $h$. Millikan (1916), who doubted Einstein's photon heuristics, completed the first measurement. After comparing his measured values against an independent determination by the Physikalisch-Technische Reichsanstalt via black body radiation, he concluded that "this value will be seen to be in exact agreement with the present photoelectric determination, which I have estimated as involving the uncertainty of $0.5 \%$ " and confirmed Einstein's predictions in every detail.

Does fixing the numerical value of universal constants have implications for the possibility of testing fundamental laws? Luckily, experimental confirmations or falsifications of a theory do not depend on the units used to express the test results. Similarly to Millikan's work, they require equality verifications.

When defining a quantity unit by fixing the numerical value of a universal constant, we also set a rule for the equality of the quantity amounts (Tal 2018). Fixing the values of $\Delta v_{\mathrm{Cs}}, c, h, e, k_{\mathrm{B}}$, and $N_{\mathrm{A}}$ we assume that the equations where they appear are true and specify the relevant equalities. Amounts are equal if the equality is consistent with Nature's laws, preserves their mathematical form, and has experimentally true consequences.

Time and frequency metrology is an example. According to Einstein (1910) "a clock is a phenomenon passing cyclically through identical phases so that we can assume that all that happens in a given cycle is identical with all that happens in an arbitrary cycle". Hence, the durations of every cycle of the microwave associated with the hyperfine transition of ${ }^{133} \mathrm{Cs}$ are stipulated to be equal, based on the underlying theoretical model.

The fixed value of the speed of light in vacuum commits us to determine as equal the lengths travelled (in a vacuum) simultaneously. Equality of masses is dictated by $h v=m c^{2}$ and, ultimately, by the equality of time intervals. As regards thermodynamics, temperature intervals are set equal by $2 h v=k_{\mathrm{B}} T$, where the mean energy per degree of freedom $k_{\mathrm{B}} T$ is expressed in terms of the Planck constant. The equality is again traced back to that of time intervals. Electrical units rely on the fixed value of the elementary charge via the Josephson and von Klitzing constants. Therefore, in addition to the equality of time intervals, their metrology takes for granted that all particles (excluding quarks) have integer charges.

There is no way to test the duration equality of caesiumclock cycles by direct measurements. However, checking if different clocks march in step, as theoretically predicted, is accessible to experimental verifications. A few additional examples are given. 
The first is the measurement of the vacuum electrical permittivity $\epsilon_{0}$ (in terms of the von Klitzing constant, $R_{\mathrm{K}}=e^{2} / h$ ) via the cross capacitance per unit length of a Thompson-Lampard capacitor, which is predicted to be $\epsilon_{0} \log (2) / \pi$ (Jackson 1999; Wood 2007). Since $\epsilon_{0}$ can also be obtained from the measured value of the fine structure constant, $\alpha=e^{2} /\left(2 \epsilon_{0} h c\right)$, the equality tests the invariance of $h / e^{2}$.

Another example is the measurement of the frequencies (in terms of $\Delta v_{\mathrm{CS}}$ ) of the $\gamma$-photons emitted by a nucleus reaching the ground state after a thermal-neutron capture. After measuring the masses (in terms of the Planck constant) of the parent and daughter nuclei, we can test the equality $m c^{2}=h v$ (Rainville et al. 2005; Jentschel and Blaum 2018).

Single electron tunnelling allows electrons to be counted one-by-one (Richardson 1997). This makes the Ohm law an equality between frequencies: a Josephson frequency, $v_{J}$, expressing the voltage and $v_{e}$, expressing the electrons per second. Hence, $R_{\mathrm{K}} v_{e} e / n_{1}=n_{2} v_{\mathrm{J}} / K_{\mathrm{J}}$ or $2 v_{e}=n_{1} n_{2} v_{\mathrm{J}}$, which equality tests the connections of the Josephson and von Klitzing constants to $h$ and $e$.

Space and time homogeneity and isotropy together with the principle of relativity imply a universal limiting velocity. Still, only the Maxwell equations identify it with the speed of light. Therefore, if the rest mass of photons is not null, the speed of light would not be identical to the limiting speed and not be universal (Guiragossian et al. (1975); Bertozzi (1964)).

As the last example, let us consider the mass measurements of the same ${ }^{28} \mathrm{Si}$ crystal by counting its atoms and using a Kibble balance (Davidson and Stock 2021). After fixing the Planck constant and contrary to the comparisons against the Pt-Ir international prototype of the kilogram, mass measurements are inertia measurements. We realise this by atom counting, which traces the mass of the ${ }^{28} \mathrm{Si}$ atom back to measurements of the electron, ${ }^{133} \mathrm{Cs}$, or ${ }^{37} \mathrm{Rb}$ masses while they emit photons and recoil.

This inertia measurement is not exactly the case when using a Kibble balance. In fact, it compares the crystal weight against a force generated by an electrical current flowing in a magnetic field. Eventually, the measured quantity is the crystal gravitational mass times the ratio between the inertial and gravitational mass of the free-falling body used to measure the acceleration due to gravity.

The equality of the two results might test the weak equivalence principle, where the crystal inertia-differently to Eötvös-like experiments, which test the universality of free fall (Nobili et al. 2013) —is sensed electromagnetically via atom count.

\section{What now?}

The elementary charge and $\Delta v_{\mathrm{CS}}$ are not fundamental constants in the same way as outlined in Sect. 2. In principle, the permeability of vacuum $\mu_{0}$ can substitute the elementary charge. However, society and industry need the practical realisation of these units with the highest possible accuracy that technology allows. The Josephson and quantum Hall effects (which link voltage and resistance to the Planck constant and elementary charge) have such a high level of accuracy and reproducibility and since 1990 they are used to realise all the electromagnetic units. Therefore, in addition to the Planck constant, the value of the elementary charge (and not $\mu_{0}$ ) has been fixed.

A system of units defined only in terms of fundamental constants, to which numerical values and dimensions are conventionally stipulated, was proposed already by Planck (1899). The gravitational constant $G$ in that case substitutes $\Delta v_{\mathrm{CS}}$ which leads to the idea of including gravity into a theory explaining the four fundamental forces as different manifestations of a single unified one. Then the mise en pratique of the units does not require atomic transitions anymore to realise the second.

\section{Conclusions}

Systems of measurement units started in history by fixing length standards, using body parts like feet or hands as reference. During the french revolution, the foundations were laid for the metric system, where natural quantities were used to establish a system of units for all time, for all people. Material prototypes were made to ensure system stability and, to assure the international unification and perfection of the metric system, the Metre Convention of 1875 set out a permanent international scientific institute at Sevres, the International Bureau of Weights and Measures (Quinn 2019).

Though the twentieth century saw a partial return to definitions based on natural standards, metrologists set up sequential calibration chains to ensure that measurement results are related to the units' primary realisations and agree within the stated uncertainties, notwithstanding the uniqueness of the material realisations of the units (Joint Committee for Guides in Metrology 2008; Stock et al. 2017).

This situation is bound to change to "parallel" implementations of traceability of measurement results to the relevant standards. The redefinition of the SI allows, in principle, anyone to implement the definitions within their required accuracy and without the need of tracing back the measurement results to unique standards owned by national metrology laboratories. 
Taking full advantage of the redefinition requires scientists not only to have a robust professional preparation but also a new scientific culture based on fundamental principles, which will allow them to build on the new system, solve complex measurement problems, and apply the newly available measurement technology for the benefit of our society.

Acknowledgements The authors thank Martin Milton (Bureau International des Poids et Mesures), Gérard Petit (Bureau International des Poids et Mesures), and Stephan Schlamminger (National Institute of Standards and Technology) for clarifying exchanges of views.

Author contributions The authors contributed equally to the manuscript.

Funding Open access funding provided by Istituto Nazionale di Ricerca Metrologica within the CRUI-CARE Agreement. This work was funded by the Ministero dell'Istruzione, dell'-Università e della Ricerca.

Availability of data and material Not applicable.

Code availability Not applicable.

\section{Declarations}

Conflict of interest The authors declare that they have no conflict of interest.

Open Access This article is licensed under a Creative Commons Attribution 4.0 International License, which permits use, sharing, adaptation, distribution and reproduction in any medium or format, as long as you give appropriate credit to the original author(s) and the source, provide a link to the Creative Commons licence, and indicate if changes were made. The images or other third party material in this article are included in the article's Creative Commons licence, unless indicated otherwise in a credit line to the material. If material is not included in the article's Creative Commons licence and your intended use is not permitted by statutory regulation or exceeds the permitted use, you will need to obtain permission directly from the copyright holder. To view a copy of this licence, visit http://creativecommons.org/licenses/by/4.0/.

\section{References}

Barbour J (2009) The nature of time. arXiv: 0903.3489

Barbour J (2001) The end of time: the next revolution in physics. Oxford University Press, Oxford

Bekenstein JD (1981) Universal upper bound on the entropy-to-energy ratio for bounded systems. Phys Rev D 23:287-298

Bertozzi W (1964) Speed and kinetic energy of relativistic electrons. Am J Phys 32(7):551-555

Bettin H, Fujii K, Man J, Mana G, Massa E, Picard A (2013) Accurate measurements of the Avogadro and Planck constants by counting silicon atoms. Ann Phys 525(8-9 SI):680-687

Clemence GM (1957) Astronomical time. Rev Mod Phys 29:2-8

Cox J, Wagman DD, Medvedev VA (1989) CODATA Key Values for Thermodynamics. Hemisphere Publishing Corp., New York, translation Andrew Motte (1729), revised Florian Cajori
Davidson S, Stock M (2021) Beginning of a new phase of the dissemination of the kilogram. Metrologia 58(3):033002

Davis RS, Milton MJT (2014) The assumption of the conservation of mass and its implications for present and future definitions of the kilogram and the mole. Metrologia 51(3):169-173

de Podesta M (2016) Rethinking the kelvin. Nat Phys 12:104-104

Einstein A (1910) Principe de relativité. Archives des sciences physiques et naturelles, vol 29, series 4 . A. Cherbuliez, Genève, pp $5-28$

Greenslade TB (1980) A striking Joule's constant determination. Phys Teach 18(3):208-209

Guiragossian Z, Rothbart G, Yearian M, Gearhart R, Murray J (1975) Relative velocity measurements of electrons and gamma rays at 15 GeV. Phys Rev Lett 34(2):335-338

Güttler B, Bettin H, Brown RJC, Davis RS, Mester Z, Milton MJT, Pramann A, Rienitz O, Vocke RD, Wielgosz RI (2019) Amount of substance and the mole in the SI. Metrologia 56(4):044002

Jackson JD (1999) A curious and useful theorem in two-dimensional electrostatics. Am J Phys 67(2):107-115

Jaynes ET (1957) Information theory and statistical mechanics. Phys Rev 106:620-630

Jentschel M, Blaum K (2018) Balancing energy and mass with neutrons. Nat Phys 14:524-524

Joint Committee for Guides in Metrology (2008) International vocabulary of metrology-basic and general concepts and associated terms (VIM), 3rd edn. Bureau International des Poids et Mesures, Sèvres

Joint Committee for Guides in Metrology (2019) The International System of Units (SI), 9th edn. Bureau International des Poids et Mesures, Sèvres

Josephson B (1962) Possible new effects in superconductive tunnelling. Phys Lett 1(7):251-253

Keller M (2018) Elementary again. Nat Phys 14:424-424

Kibble B (1976) A measurement of the gyromagnetic ratio of the proton by the strong field method. In: Sanders J, Wapstra A (eds) Atomic masses and fundamental constants 5. Springer, Boston, pp 545-551

Lan SY, Kuan PC, Estey B, English D, Brown JM, Hohensee MA, Müller H (2013) A clock directly linking time to a particle's mass. Science 339(6119):554-557

Lévy-Leblond JM (1977) On the conceptual nature of the physical constants. La Rivista del Nuovo Cimento 7(2):187-214

Mana G, Massa E (2012) The Avogadro and the Planck constants for redefinition of the kilogram. Rivista del Nuovo Cimento 35(7):353-388

Massa E, Mana G (2016) Counting atoms. Nat Phys 12:522-522

Millikan RA (1916) A direct photoelectric determination of Planck's “" $h$ '. Phys Rev 7:355-388

Milton MJT (2011) A new definition for the mole based on the Avogadro constant: a journey from physics to chemistry. Philos Trans R Soc Lond A Math Phys Eng Sci 369(1953):3993-4003

Milton MJT, Mills IM (2009) Amount of substance and the proposed redefinition of the mole. Metrologia 46(3):332-338

Mohr PJ, Newell DB, Taylor BN, Tiesinga E (2018) Data and analysis for the CODATA 2017 special fundamental constants adjustment. Metrologia 55(1):125-146

Müller H (2014) Quantum mechanics, matter waves, and moving clocks. In: Tino GM, Kasevich MA (eds) proceedings of the International School of Physics "Enrico Fermi" 2013, Course 188-Atom Interferometry, IOS Press, Amsterdam, pp 339-418

Newton I (1689) Philosophiae naturalis principia mathematica, Bk. 1. University of California Press, Berkeley, 1934, translation Andrew Motte (1729), revised Florian Cajori

Nobili AM, Lucchesi DM, Crosta MT, Shao M, Turyshev SG, Peron R, Catastini G, Anselmi A, Zavattini G (2013) On the universality of 
free fall, the equivalence principle, and the gravitational redshift. Am J Phys 81(7):527-536

Perrin JB (1909) Mouvement brownien et réalité moléculaire. Ann Chim Phys VII I(3):5-114

Planck M (1899) Über irreversible Strahlungsvorgänge. Sitzungsberichte der Königlich Preußischen Akademie der Wissenschaften zu Berlin 1:440-480

Quinn T (2019) The metre convention of 1875: a commentary and new English edition. La Rivista del Nuovo Cimento 42(6):261-300

Rainville S, Thompson JK, Myers EG, Brown JM, Dewey MS, Kessler EGJ, Deslattes RD, Börner HG, Jentschel M, Mutti P, Pritchard DE (2005) A direct test of $E=m c^{2}$. Nature 438(5656):1096-1097

Richardson WH (1997) Possibility of a single electron tunneling diode and a controllable saturated tunneling current. Appl Phys Lett 71(8):1113-1115

Rovelli c (1996) Relational quantum mechanics. Int J Theor Phys 35(8):1637-1678

Schlamminger S, Haddad D (2019) The Kibble balance and the kilogram. Comptes Rendus Physique 20(1):55-63

Shannon CE (1948) A mathematical theory of communication. Bell Syst Tech J 27(3):379-423

Soffel M, Klioner SA, Petit G, Wolf P, Kopeikin SM, Bretagnon P, Brumberg VA, Capitaine N, Damour T, Fukushima T, Guinot B, Huang TY, Lindegren L, Ma C, Nordtvedt K, Ries JC, Seidelmann PK, Vokrouhlick D, Will CM, Xu C (2003) The IAU 2000 resolutions for astrometry, celestial mechanics, and metrology in the relativistic framework: explanatory supplement. Astron J 126(6):2687-2706

Stephenson A, la Piana L, Mulville D, Rutledge P, Bauer F, Folta D, Greg A, Sackheim R, Norvig P (1999) Mars climate orbiter mishap investigation board phase I report. NASA, November 10, 1999

Stock M, Davidson S, Fang H, Milton M, de Mirandés E, Richard P, Sutton C (2017) Maintaining and disseminating the kilogram following its redefinition. Metrologia 54(6):S99-S107

Stock M, Davis R, de Mirandés E, Milton MJT (2019) The revision of the SI-the result of three decades of progress in metrology. Metrologia 56(2):022001

Tait P (1884) Note on reference frames. Proc R Soc Edinb 12:743-745

Tal E (2018) Naturalness and convention in the international system of units. Measurement 116:631-643

von Klitzing K (2017) Metrology in 2019. Nat Phys 13:198-198

von Klitzing K, Dorda G, Pepper M (1980) New method for highaccuracy determination of the fine-structure constant based on quantized Hall resistance. Phys Rev Lett 45:494-497

Witkin R (1983) Jet's fuel ran out after metric conversion errors. The New York Times (July 30, 1983)

Wood B (2007) The NRC calculable capacitor and its role in the SI. NCSLI Meas 2(1):86-90

Publisher's Note Springer Nature remains neutral with regard to jurisdictional claims in published maps and institutional affiliations. 